\title{
Fractals for minimalists
}

\section{Mihály Bessenyei and Evelin Pénzes}

Dedicated to the 95th birthday of Professor János Aczél.

\begin{abstract}
The aim of this note is to present an elementary way to fractals which completely avoids advanced analysis and uses purely naive set theory. The approach relies on fixed point methods, where the role of the Banach contraction principle is replaced by a slightly improved version of the Knaster-Tarski fixed point theorem.
\end{abstract}

Mathematics Subject Classification. Primary 28A80; Secondary 03E20, 03E75.

Keywords. Fractal, Invariance equation, Isotone map, Fixed point theorem.

\section{Motivations}

Although fractals definitely belong to the most popular mathematical inventions, they have no unified definition in the technical literature. Measure theorists prefer to capture their fragmented nature. Mandelbrot himself considered them as sets for which the Hausdorff-Besicovitch dimension strictly exceeds the topological dimension (see his pioneer work [7], or its English version [8]).

Fixed point theorists focus on self-similarity, which is the other characteristic property of fractals. This approach and its interaction with the HausdorffBesicovitch dimension was elaborated by Hutchinson [4]. In the forthcoming investigations, we also follow this path.

Throughout this note, $X$ stands for an arbitrary set, and $\mathscr{P}(X)$ denotes its power set. Assume that $\mathscr{T}=\left\{T_{\gamma}: F(X) \rightarrow \mathscr{F}(X) \mid \gamma \in \Gamma\right\}$ is a nonempty family of maps, where $\mathscr{F}(X)$ is a subfamily of $\mathscr{P}(X)$. We say that $H \in \mathscr{F}(X)$ is a $\mathscr{T}$-fractal if it fulfills the invariance equation

This paper was supported by the Jànos Bolyai Research Scholarship of the Hungarian Academy of Sciences, by the ÚNKP-18-2 New National Excellence Program of the Ministry of Human Capacities, and by the ÚNKP-19-4 New National Excellence Program of the Ministry of Innovation and Technology. 


$$
H=\bigcup_{\gamma \in \Gamma} T_{\gamma}(H)
$$

The family $\mathscr{T}$ is frequently called iterated function system (IFS), while fractals are termed the attractors of the underlying IFS. To develop a satisfactory fractal theory, that is, a theory in which fractals are uniquely defined objects via their invariance equation, the family $\mathscr{F}(X)$ and the mapping class $\mathscr{T}$ have to be declared.

In the classical setting, $\mathscr{F}(X)$ is the family of nonempty, bounded, closed subsets of a complete metric space, and $\mathscr{T}$ consists of finitely many contractions. Then, Hutchinson's fundamental result [4] guarantees the unique resolvability of (1). His brilliant approach interprets the invariance equation as a fixed point problem, which has exactly one solution in the complete metric space of fractals according to the Blaschke Theorem and the Banach Contraction Principle.

Later Mandelbrot proposed to use 'fractal' without a pedantic definition, since we can see fractals everywhere [2]. Motivated by this philosophy, we take the liberty to consider fractals everything: We will not distinguish $\mathscr{F}(X)$ and $\mathscr{P}(X)$. The family $\mathscr{T}$ will consist of arbitrarily many isotone or continuous maps (their precise definition is given in the next section). Then, a slightly improved version of the Knaster-Tarski theorem [6] guarantees a smallest fractal fulfilling (1).

Our approach provides a minimalists' fractal theory in a double sense: a minimal theoretical setup leads to the minimum solution of the invariance equation. This elementary way completely avoids the advanced tools of analysis, and simultaneously reflects the method and the beauty of the fixed point approach.

\section{Isotone and continuous maps}

A mapping $T: \mathscr{P}(X) \rightarrow \mathscr{P}(X)$ is called isotone, if it preserves the settheoretic inclusion, that is $T(A) \subseteq T(B)$ holds provided that $A \subseteq B$. Isotone maps have several obvious properties. For instance, the images of the emptyset and the basic set under an isotone map $T$ are

$$
T(\emptyset)=\bigcap\{T(A) \mid A \in \mathscr{P}(X)\} \quad \text { and } \quad T(X)=\bigcup\{T(A) \mid A \in \mathscr{P}(X)\} .
$$

Note also, that isotone maps are superadditive with respect to union, while subadditive with respect to intersection. That is, for any isotone map $T: \mathscr{P}(X) \rightarrow$ $\mathscr{P}(X)$, we have

$$
\bigcup_{A \in \mathscr{A}} T(A) \subseteq T(\bigcup \mathscr{A}) \text { and } T(\bigcap \mathscr{A}) \subseteq \bigcap_{A \in \mathscr{A}} T(A) .
$$


An other important mapping class (which will turn out to be a subfamily of isotone maps) is the class of continuous maps. In the power set $\mathscr{P}(X)$, it is useful to regard countable chains as sequences and the union of the chain as the limit. Guided by this, a map $T: \mathscr{P}(X) \rightarrow \mathscr{P}(X)$ is said to be continuous, if, for every countable chain $\mathscr{L}$,

$$
T(\bigcup \mathscr{L})=\bigcup_{L \in \mathscr{L}} T(L)
$$

Further obvious properties will play a distinguished role in our investigations. Therefore we formulate them as Propositions. The first one highlights the precise relation of isotone and continuous self-maps, while the second and third ones show how to generate isotone and continuous maps using these kind of maps.

Proposition 1. Any continuous self-map of a power set is isotone. Furthermore, any self-map of a finite power set is isotone if and only if it is continuous.

Proof. Let $T: \mathscr{P}(X) \rightarrow \mathscr{P}(X)$ be a continuous map, and assume that $A \subseteq B$. Consider the chain $\mathscr{L}=\{A, B\}$. Then,

$$
T(A) \subseteq T(A) \cup T(B)=\bigcup_{L \in \mathscr{L}} T(L)=T(\bigcup \mathscr{L})=T(B),
$$

resulting in the first statement. To prove the second one, it suffices to prove only that any isotone map $T$ on a finite power set is continuous. Observe first that, any countable chain in a finite set is finite. For a finite chain $\mathscr{L}$, the set $\{T(L) \mid L \in \mathscr{L}\}$ is a chain again, since $T$ is isotone. Moreover, if $L$ represents the greatest member of $\mathscr{L}$, then $T(L)$ becomes the greatest member in the corresponding chain. Therefore, $T(\bigcup \mathscr{L})=T(L)=\bigcup T(\mathscr{L})$ holds, yielding continuity.

Proposition 2. The finite composition of isotone (respectively, continuous) maps is isotone (respectively, continuous). In particular, the iterates of an isotone (respectively, continuous) map is also isotone (respectively, continuous).

Proposition 3. If $\mathscr{T}:=\left\{T_{\gamma}: \mathscr{P}(X) \rightarrow \mathscr{P}(X) \mid \gamma \in \Gamma\right\}$ is an isotone (respectively: continuous) family and $T: \mathscr{P}(X) \rightarrow \mathscr{P}(X)$ is defined by

$$
T(H)=\bigcup_{\gamma \in \Gamma} T_{\gamma}(H),
$$

then $T$ is also isotone (respectively, continuous).

Proof. We restrict the proof only to the second statement. Let $\mathscr{L}$ be a countable chain. Then, by the continuity of the members, 
$T(\bigcup \mathscr{L})=\bigcup_{\gamma \in \Gamma} T_{\gamma}\left(\bigcup_{L \in \mathscr{L}} L\right)=\bigcup_{\gamma \in \Gamma} \bigcup_{L \in \mathscr{L}} T_{\gamma}(L)=\bigcup_{L \in \mathscr{L}} \bigcup_{\gamma \in \Gamma} T_{\gamma}(L)=\bigcup_{L \in \mathscr{L}} T(L)$.

This verifies the continuity of $T$.

A nontrivial property of isotone maps, the well-known result of Knaster and Tarski [6], states that each isotone map has a fixed point. The next theorem slightly improves this statement, guaranteeing the existence of a smallest fixed point. Hence, in this form, it may be considered as an existence and uniqueness result. Adopting the idea of Kantorovitch [5], the smallest fixed point can even be represented with the help of the orbit of the empty set. In the sequel, $\operatorname{Fix}(T)$ shall stand for the set of fixed points of $T$.

Theorem 1. If $T: \mathscr{P}(X) \rightarrow \mathscr{P}(X)$ is isotone, then $\operatorname{Fix}(T)$ is nonempty, and $\bigcap \operatorname{Fix}(T)$ is the smallest fixed point of $T$. Moreover, if $T$ is continuous, then the smallest fixed point can be represented as

$$
\bigcup_{n \in \mathbb{N}} T^{n}(\emptyset)=\bigcap \operatorname{Fix}(T)
$$

Proof. By the Knaster-Tarski fixed point theorem, Fix $(T)$ is nonempty. Let $H \in \operatorname{Fix}(T)$ be arbitrary and define $H_{0}:=\bigcap \operatorname{Fix}(T)$. Then $H_{0} \subseteq H$, and hence, $T\left(H_{0}\right) \subseteq T(H)=H$ holds for all members of $\operatorname{Fix}(T)$. Thus $T\left(H_{0}\right) \subseteq H_{0}$, yielding that $T$ maps the power set $\mathscr{P}\left(H_{0}\right)$ into itself. By the Knaster-Tarski fixed point theorem again, $T$ has a fixed point which is a subset of $H_{0}$. This can occur only if this fixed point is $H_{0}$ itself. Therefore $H_{0}$ is the smallest fixed point of $T$.

Consider now the family

$$
\mathscr{L}:=\left\{T^{n}(\emptyset) \mid n \in \mathbb{N}\right\} .
$$

For an isotone map $T$, the trivial inclusion $\emptyset \subseteq T(\emptyset)$ implies $T(\emptyset) \subseteq T^{2}(\emptyset)$. Iterating this process, one can conclude that $\mathscr{L}$ is an increasing chain. On the other hand, using now $\emptyset \subseteq H_{0}$ and Proposition 2, $T^{n}(\emptyset) \subseteq T^{n}\left(H_{0}\right)=H_{0}$ follows for all $n \in \mathbb{N}$. Therefore,

$$
\bigcup_{n \in \mathbb{N}} T^{n}(\emptyset)=\bigcup \mathscr{L} \subseteq H_{0}=\bigcap \operatorname{Fix}(T)
$$

always holds for purely isotone maps. Assume now that our map is continuous. By Proposition 1, the just proved facts above remain true for continuous $T$, as well. Moreover, by continuity,

$$
T(\bigcup \mathscr{L})=\bigcup_{L \in \mathscr{L}} T(L)=\bigcup_{n \in \mathbb{N}} T^{n+1}(\emptyset)=\bigcup_{n \in \mathbb{N}} T^{n}(\emptyset)=\bigcup \mathscr{L} .
$$

This verifies that $\bigcup \mathscr{L}$ is a fixed point for $T$. However, $H_{0}$ is the smallest fixed point. This implies the reverse inclusion $H_{0} \subseteq \bigcup \mathscr{L}$. 
Observe that (3) holds for such isotone maps that satisfy the continuity property only on the special chain $\mathscr{L}=\left\{T^{n}(\emptyset) \mid n \in \mathbb{N}\right\}$. Though this observation is valid for the forthcoming results, we shall formulate them under the assumption of continuity.

Finally, we present some examples of isotone and continuous maps and describe their fixed point properties, as well. The details of the proofs are left to the reader.

Example 1. If $A \subseteq X$ is arbitrary, then the map $T: \mathscr{P}(X) \rightarrow \mathscr{P}(X)$ defined by $T(H)=A$ is continuous. Its smallest and greatest fixed point is $A$.

Example 2. The identity map of any power set is continuous, and each subset is a fixed point. The smallest fixed point is the emptyset, while the greatest one is the basic set.

Example 3. Let $X$ be an infinite set, and define $T: \mathscr{P}(X) \rightarrow \mathscr{P}(X)$ in the following way. Let $T(H)=\emptyset$ if $H$ is finite and $T(H)=X$ if $H$ is infinite. Then $T$ is an isotone, but not continuous map. Its smallest fixed point is the emptyset, while the greatest one is $X$. No more fixed point exists.

Example 4. Let $X$ be a vector space, and fix $x_{0} \in X$ and $\lambda \in \mathbb{R}$ arbitrarily. Then the mapping $T: \mathscr{P}(X) \rightarrow \mathscr{P}(X)$ given by $T(H)=\left\{\lambda x+x_{0} \mid x \in\right.$ $H\} \cup\left\{x_{0}\right\}$ is continuous, and its smallest fixed point $H_{0}$ can be represented as

$$
H_{0}=\left\{\left(\sum_{k=1}^{n} \lambda^{k-1}\right) x_{0} \mid n \in \mathbb{N}\right\} .
$$

Example 5. Let $f: X \rightarrow Y$ be an arbitrary function, and define the map $T_{f}: \mathscr{P}(X) \rightarrow \mathscr{P}(Y)$ via the standard way of "image of sets"

$$
T_{f}(H):=\{f(x) \mid x \in H\} .
$$

Then, $T_{f}$ is continuous.

Observe, that Example 5 extends the first statement of Example 4. Note also, that the representation of the smallest fixed point in Example 4 follows directly from $(3)$.

\section{The main result}

Now we are in the position to formulate and prove the main result and highlight its connection with classical fractal theory. We illustrate this connection with the help of well-known fractals.

Theorem 2. If $\mathscr{T}$ is a family of isotone maps, then there exists a smallest $\mathscr{T}$ fractal. Moreover, if $\mathscr{T}$ is a continuous family, then this fractal is obtained via (3). 
Proof. Let $\mathscr{T}:=\left\{T_{\gamma}: \mathscr{P}(X) \rightarrow \mathscr{P}(X) \mid \gamma \in \Gamma\right\}$ and define $T: \mathscr{P}(X) \rightarrow$ $\mathscr{P}(X)$ with (2). By Proposition 3, $T$ is isotone (respectively: continuous) provided that $\mathscr{T}$ is an isotone (respectively: continuous) family. Hence the statements follow immediately from Theorem 1 .

Classical fractals can also be studied in the minimalists' framework as the first Corollary shows. Its first statement is a consequence of Example 5, Proposition 3 and Theorem 2, the second one is obvious. Details of the proof are omitted.

Corollary 1. If $f_{1}, \ldots, f_{n}$ are contractions of a complete metric space, then the invariance equation

$$
H=T_{f_{1}}(H) \cup \cdots \cup T_{f_{n}}(H)
$$

has a smallest solution, which is a subset of the corresponding nonempty, closed, bounded one.

It may occur, that the minimalist solution of the classical invariance equation is the emptyset. However, we can avoid trivial cases by adding some extra parts to the original equation. Moreover, it can be done in such a way, that the modification will have no effect on the original nonempty, closed, bounded solution. The next Corollaries explain this phenomenon.

Corollary 2. The smallest real fractal $H_{0}$ which satisfies the invariance equation

$$
H=\frac{1}{3} H \cup\left(\frac{1}{3} H+\frac{2}{3}\right) \cup\{0\}
$$

is a proper subset of the Cantor-set, and consists of exactly those real numbers whose ternary expansions are finite, and the digits belong to the set $\{0,2\}$.

Hint. By Example 4, the right-hand side of the invariance equation above contains continuous maps. Therefore, by Theorem 2, there exists a smallest fractal satisfying the invariance equation, indeed. Using induction and the fact $T(\emptyset)=\{0\}$, one can easily prove that

$$
T^{n+1}(\emptyset)=\left\{\left(0, x_{1} \ldots x_{n}\right)_{3} \mid x_{k} \in\{0 ; 2\}, k=1, \ldots, n\right\},
$$

where $(\cdot)_{3}$ stands for the basic ternary expansion of reals. Hence (3) reduces to

$$
H_{0}=\left\{\left(0, x_{1} \ldots x_{n}\right)_{3} \mid x_{k} \in\{0 ; 2\}, k=1, \ldots, n ; n \in \mathbb{N}\right\},
$$

as it was stated. The Cantor-set itself is a solution (more precisely, the unique nonempty, closed, bounded one) of the invariance equation above. Therefore, by Theorem 2, $H_{0}$ is a subset of the Cantor-set. On the other hand, $H_{0}$ is nonempty and countable, so it is a proper subset. 
Corollary 3. For all $i, j \in\{0,1,2\}$, define the mappings $T_{i, j}: R \rightarrow \mathbb{R}$ by

$$
T_{i j}(x, y)=\left(\frac{x+i}{3}, \frac{y+j}{3}\right) .
$$

The smallest planar fractal which satisfies the invariance equation

$$
H=\bigcup\left\{T_{i j}(H) \mid i, j \in\{0,1,2\},(i, j) \neq(1,1)\right\} \cup\{(0,0)\}
$$

is a proper subset of the Sierpinski-carpet, and consists of exactly those points whose components' ternary expansions are finite, and have no position in which both numbers have the digit 1.

Corollary 4. For all $i, j \in\{0,1,2,3\}$, define the mappings $T_{i, j, k}: R \rightarrow \mathbb{R}$ by

$$
T_{i j k}(x, y, z)=\left(\frac{x+i}{3}, \frac{y+j}{3}, \frac{z+k}{3}\right) .
$$

The smallest $3 D$ fractal which satisfies the invariance equation

$H=\bigcup\left\{T_{i j k}(H) \mid i, j, k \in\{0,1,2\},(1,1) \notin\{(i, j),(j, k),(k, i)\}\right\} \cup\{(0,0,0)\}$

is a proper subset of the Menger-sponge, and consists of exactly those points whose components' ternary expansions are finite, and have no position in which each number has the digit 1.

\section{Some historical comments}

The joint result of Knaster and Tarski [6] is the source of order theoretic fixed point results. It appeared in a conference report of the meeting of the Warsaw Division of the Polish Mathematical Society. This report does not contain the proof, and states only the existence of a fixed point. As applications, the Banach Mapping Theorem [1] and the Schröder-Bernstein Theorem are mentioned. Then, Tarski developed this result alone. In the second footnote of a later article [11], he commemorates this as follows:

In 1927 Knaster and the author proved a set-theoretical fixpoint theorem by which every function, on and to the family of all subsets of a set, which is increasing under set-theoretical inclusion has at least one fixpoint; see [6], where some applications of this result in set theory (a generalization of the Cantor-Bernstein theorem) and topology are also mentioned. A generalization of this result is the lattice-theoretical fixpoint theorem stated above. The theorem in its present form and its various applications and extensions were found by the author in 1939 and discussed by him in a few public lectures in 1939-1942. 
The fundamental paper of Tarski [11] presents two fixed point theorems for isotone maps of complete lattices. The first one extends considerably the original result of Knaster and Tarski, and claims the existence of smallest and greatest fixed points. The second theorem formulates an analogous statement for a commuting family of isotone maps. Then several applications are given, among others, generalizations of the Weierstrass Theorem and the CantorBendixson Theorem. Results in the theory of Boolean algebras are also presented.

Consider the union of the upper half-fixed points and the intersection of the lower half-fixed points of an isotone map $T: \mathscr{P}(X) \rightarrow \mathscr{P}(X)$ :

$$
\begin{aligned}
\mathscr{A} & :=\{H \subseteq X \mid H \subseteq T(H)\}, & & A:=\cup \mathscr{A} ; \\
\mathscr{B} & :=\{H \subseteq X \mid T(H) \subseteq H\}, & & B:=\cap \mathscr{B} .
\end{aligned}
$$

The standard proof of the Knaster-Tarski Theorem, for example, as Shapiro [9] presents, is to show that $A$ is a fixed point of $T$. This proof can directly be adopted to show the fixed point property of $B$. In fact, the first proof shows the existence of the greatest, while the second one the existence of the smallest fixed point implicitly.

The famous Hungarian journal, the Mathematical and Physical Journal for Secondary Schools, posed problems called 'hors concours' between 1973 and 1984. One of them, labeled P. 329, was the Knaster-Tarski Theorem. Only three solutions arrived, and two of them were correct. The presented solution follows the nonstandard way [10], showing that $B$ (see above) is a fixed point. The problem itself does not claim the minimum property.

Of course, our set-theoretic approach in itself is not sufficient to show the nice geometrical properties of fractals. However, as we have pointed out in the motivations, at least it opens a window to the role of fixed point theory. Therefore, we hope, this note will have some didactic impact. For those who are interested in fixed point theory and its applications, we refer to the works of Granas and Dugundji [3], of Shapiro [9] and of Zeidler [12].

\section{Acknowledgements}

Open access funding provided by University of Debrecen (DE). The authors wish to express their gratitude to ERIKA ANDIRKó, who helped to find the papers of Tarski, to GYUlA MAKSA, who translated the original French texts, and to ÁrPÁD SzÁz, who gave a lot of helpful advice. 
Open Access. This article is distributed under the terms of the Creative Commons Attribution 4.0 International License (http://creativecommons.org/licenses/by/4.0/), which permits unrestricted use, distribution, and reproduction in any medium, provided you give appropriate credit to the original author(s) and the source, provide a link to the Creative Commons license, and indicate if changes were made.

Publisher's Note Springer Nature remains neutral with regard to jurisdictional claims in published maps and institutional affiliations.

\section{References}

[1] Banach, S.: Un théorème sur les transformations biunivoques. Fund. Math. 6, 236-239 (1924)

[2] Barnsley, M.: Fractals Everywhere. Academic Press, Inc., Boston (1988)

[3] Granas, A., Dugundji, J.: Fixed Point Theory. Springer Monographs in Mathematics. Springer, New York (2003)

[4] Hutchinson, J.E.: Fractals and self-similarity. Indiana Univ. Math. J. 30(5), 713-747 (1981)

[5] Kantorovitch, L.: The method of successive approximations for functional equations. Acta Math. 71, 63-97 (1939)

[6] Knaster, B., Tarski, A.: Un théoreme sur lesfonctions d'ensembles. Ann. Soc. Pol. Math. 6, 133-134 (1927)

[7] Mandelbrot, B.: Les objets fractals. Flammarion, Editeur, Paris (1975). Forme, hasard et dimension, Nouvelle Bibliothèque Scientifique

[8] Mandelbrot, B.: Fractals: Form, Chance, and Dimension, revised ed., W. H. Freeman and Co., San Francisco (1977). Translated from the French

[9] Shapiro, J.H.: A fixed-point farrago. In: Axler, S., Casacuberta, C., MacIntyre, A., Ribet, K., Sabbah, C., Süli, E., Woyczyński, W.A. (eds.) Universitext, Springer, Berlin (2016)

[10] Szegedy, P.: Solution to problem P.329. KöMaL Math. Phys. J. Second. Sch. 61(2), 75 (1980)

[11] Tarski, A.: A lattice-theoretical fixpoint theorem and its applications. Pac. J. Math. 5, 285-309 (1955)

[12] Zeidler, E.: Nonlinear Functional Analysis and Its Applications. I. Springer, New York (1986) Fixed-point theorems, Translated from the German by Peter R. Wadsack

Mihály Bessenyei and Evelin Pénzes

Institute of Mathematics

University of Debrecen

Debrecen PF. 124010

Hungary

e-mail: besse@science.unideb.hu

Evelin Pénzes

e-mail: penzesevelyn@gmail.com

Received: April 2, 2019

Revised: August 27, 2019 